\title{
La technique à visage humain
}

\section{Tim Hunkin}

\section{OpenEdition \\ Journals}

Édition électronique

URL : https://journals.openedition.org/tc/8472

DOI : $10.4000 /$ tc. 8472

ISSN : 1952-420X

\section{Éditeur}

Éditions de l'EHESS

\section{Édition imprimée}

Date de publication : 6 juin 2017

Pagination : 64-81

ISBN : 978-2-7132-2707-3

ISSN : 0248-6016

Référence électronique

Tim Hunkin, «La technique à visage humain », Techniques \& Culture [En ligne], 67 | 2017, mis en ligne le 06 juin 2019, consulté le 29 septembre 2022. URL : http://journals.openedition.org/tc/8472 ; DOI : https://doi.org/10.4000/tc.8472 


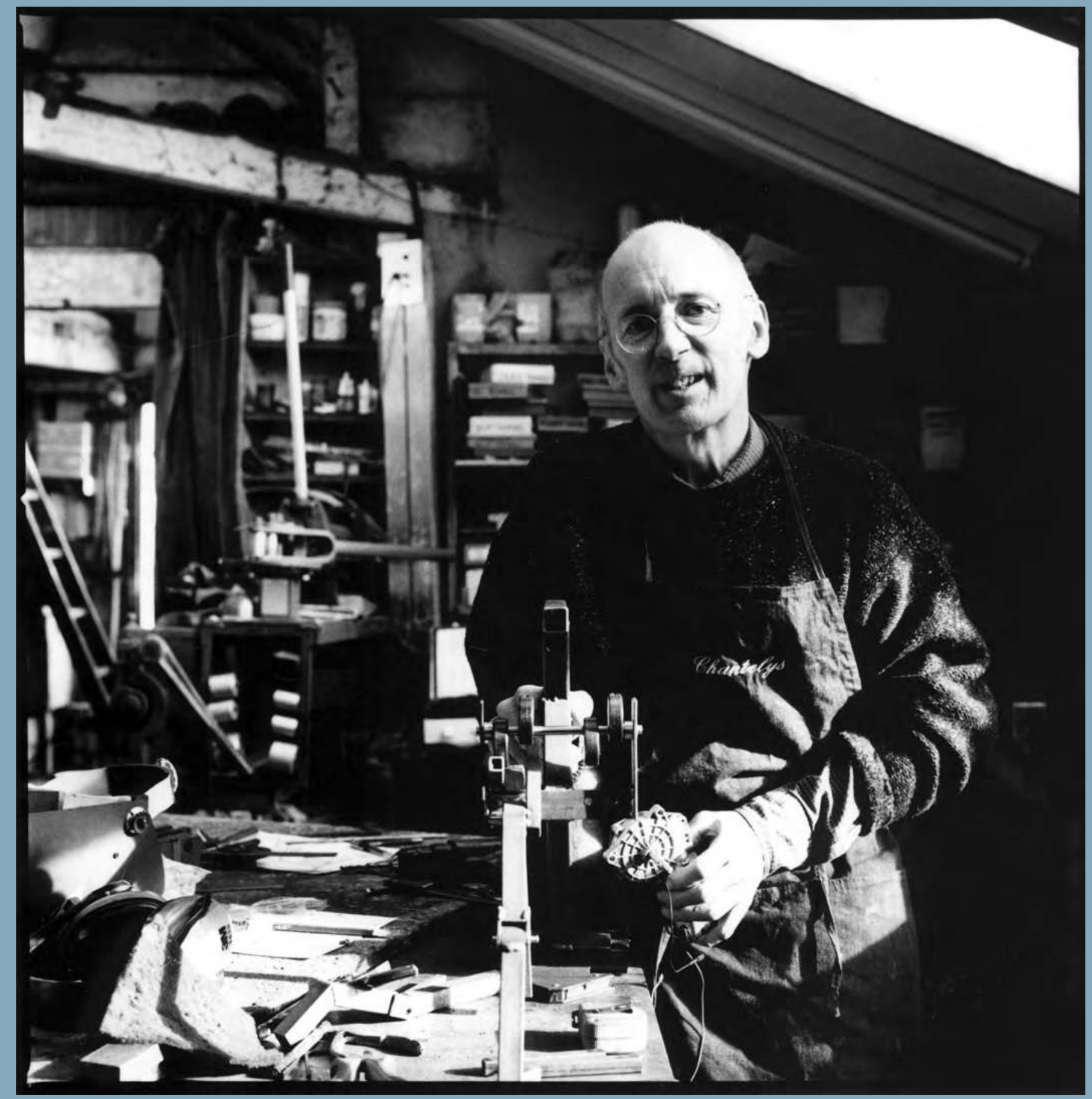




\section{La technique à visage humain "Technology is what makes us human "}

Il n'est pas courant d'entendre quelqu'un s'excuser d'arriver en retard pour une conférence, parce qu'il lui fallait terminer en toute hâte une cage pour les tigres du Zoo de Londres! Mais il est vrai que Tim Hunkin appartient à une espèce rare : celle de ces créateurs géniaux pour qui la fabrication d'automates représente seulement un passetemps parmi d'autres. Al Jazari et Albert le Grand tout comme Léonard de Vinci, Vaucanson ou Gaston Lagaffe appartiennent aussi à ce club très fermé. Alors que chacun d'entre eux s'est pas mal débrouillé dans son genre, seul le dernier peut rivaliser véritablement avec Tim Hunkin par l'humour de ses créations ${ }^{1}$. Aussi ne s'étonnera-t-on pas d'apprendre qu'après ses études d'ingénieur à Cambridge, il ait gagné sa vie en faisant, par exemple, des caricatures pour un grand hebdomadaire anglais (The Observer), des émissions scientifiques extrêmement populaires à la télévision (The Secret Life of Machines TV Series) ou encore des éléments de scénographie pour le Musée de la science à Londres. Et l'on n'oubliera pas non plus la manière dont il a fabriqué, au cours des années, de merveilleuses horloges dont certaines rivalisent sans difficulté avec celles qui ornent les cathédrales et les beffrois de nos anciennes cités, que ce soit par la diversité de leurs sources d'énergie, la sophistication de leurs mécanismes ou le jeu de leurs automates. C'est pourtant dans un autre domaine, encore, que celui-ci donne véritablement toute la mesure de sa fantaisie et de son ingéniosité mécanique. Tim Hunkin s'est visiblement fixé comme mission de révéler à un monde qui l'ignore, le potentiel créatif mais aussi vaguement subversif et progressiste des machines à sous ; un potentiel d'autant plus négligé que ces dernières ont surtout été utilisées jusqu'alors pour captiver et exploiter les gogos. 
On connaît trop d'artistes dont le progressisme affiché n'a d'égal que l'empressement avec lequel ils s'efforcent de séduire par ce biais les élites installées qui gouvernent le milieu de l'art : directeurs de galeries célèbres, commanditaires fortunés ou conservateurs de musées. À l'inverse de ces derniers, Tim Hunkin a su découvrir un moyen authentiquement démocratique de vivre de son art : il le fait, en effet, en récoltant chaque jour, les pièces de monnaie accumulées dans les extraordinaires machines à sous surréalistes qu'il fabrique lui-même et qu'il renouvelle régulièrement dans les différents lieux où elles sont exposées, pour le plus grand plaisir d'un public charmé par son impertinence et par son inventivité. C'est d'ailleurs en utilisant ce procédé qu'il a découvert un moyen ingénieux de fournir une source de revenu complémentaire à des hôpitaux ou à des musées; et même si cet apport reste modeste, il n'en a pas moins le mérite d'apporter aussi un peu de poésie et de bonne humeur en des lieux qui en ont bien besoin.

Mais plutôt que de gloser plus longtemps sur Tim Hunkin et sur son œuvre multiforme, mieux vaut lui laisser la parole. D'autant que lorsqu'il s'agit de défendre la vocation qui l'anime, ce créateur de caractère pourtant profondément modeste ne coupe pas les cheveux en quatre et ne mâche pas non plus ses mots.

Denis Vidal

Je viens de terminer une horloge pour le zoo de Londres avec toutes sortes d'automates et un mouvement relativement compliqué. Les ingénieurs me considèrent généralement comme un artiste ; ce n'est pas complètement faux mais la vérité est que je passe la plus grande partie de mon temps à résoudre des problèmes que l'on peut qualifier de problèmes d'ingénieurs. D'ailleurs le computer et les mécanismes pneumatiques que j’ai employés pour cette horloge sont utilisés pour des automates industriels. Une des gageures avec cette horloge était qu'elle soit vraiment fiable parce que personne ne sera là, par la suite, pour la contrôler. Et il faut aussi qu'elle réponde à toutes les normes de sécurité en vigueur.

J'ai fait des études d'ingénieur, il y a longtemps, au niveau le plus théorique. Mais je suis de plus en plus convaincu que le travail de l'ingénieur est d'abord un art, même au niveau des analyses les plus sophistiquées. Les écoles d'ingénieur se vantent du raffinement théorique de leur méthode, inspirées des sciences exactes, ou bien d'utiliser la réalité virtuelle. En revanche, elles se désintéressent complètement de tout ce qui fait penser à du bricolage. Je pense, à l'opposé, qu'une de nos plus grandes capacités - en tant qu'êtres humains - réside précisément à savoir « penser avec nos mains » et que c'est une grave erreur de dédaigner la dimension intuitive du travail d'ingénieur comme si cela en représentait une étape dépassée.

Francis Evans est mort, il y a deux ans. Il était professeur d'engineering à l'université Hallam Il de Sheffield et il avait une vraie passion pour l'histoire de la technique. C'était un de mes héros. Il est célèbre pour avoir créé une sorte d'arche que l'on trouve aujourd'hui pratiquement dans 


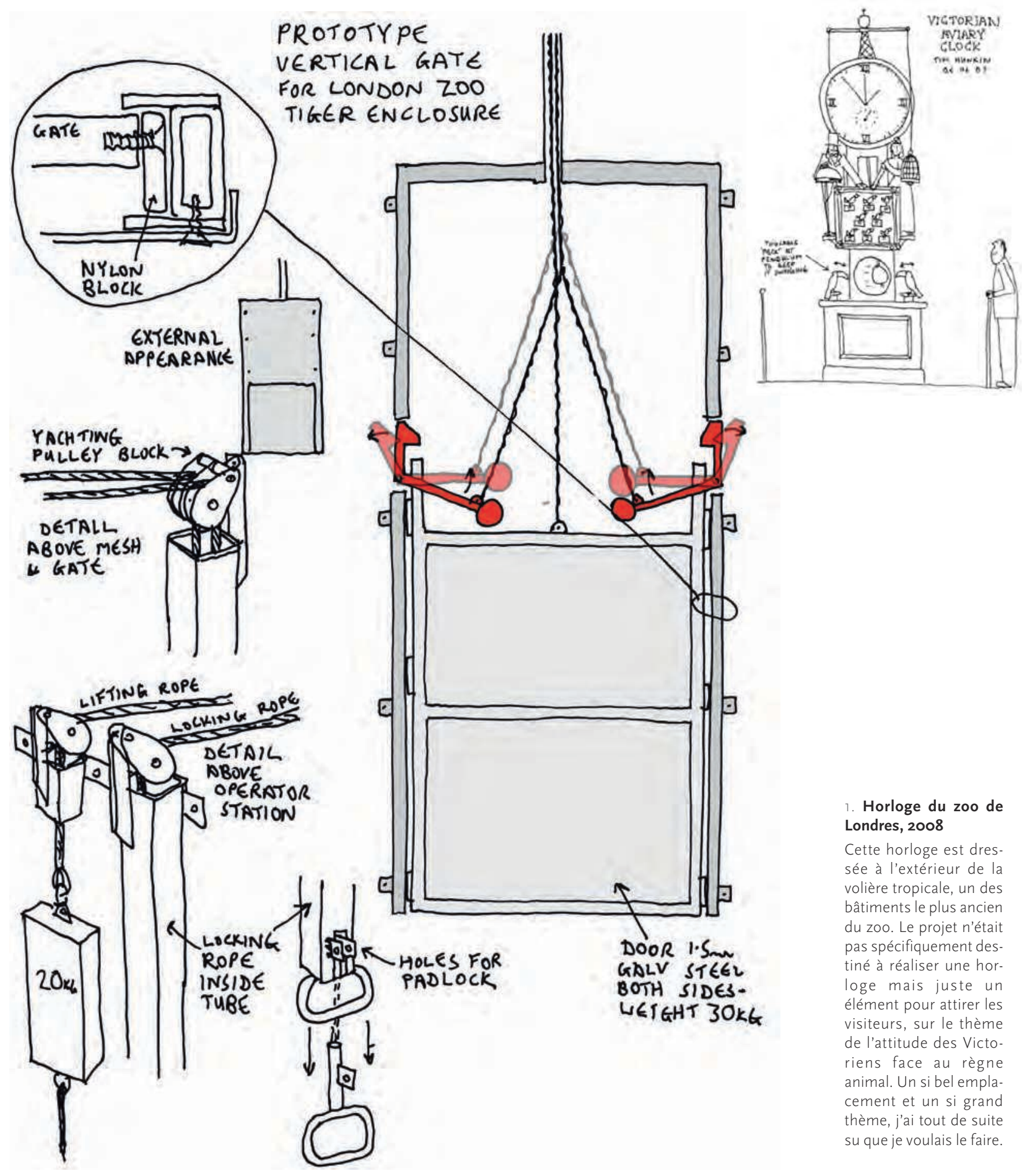


tous les musées de science au monde - on se sert d'un bloc en forme de demi-cercle pour disposer dessus des blocs amovibles qui définissent une arche. Puis une fois cette arche assemblée, on enlève le bloc sur lequel l'arche a été édifiée; et, bien que les blocs ne soient pas fixés entre eux, elle est suffisamment solide pour que l'on puisse marcher dessus. Mais si Francis est vraiment mon héros, c'est d'abord à cause d'un de ses écrits qui s'intitule : "Two legs, thing using and talking ». Ce texte a non seulement radicalement changé la manière dont j'envisage la technique mais aussi la perception que je peux avoir de moi-même.

Son idée-force consiste à penser que la technique n'est pas quelque chose qui nous est extérieure, mais bien une dimension fondamentale de la nature humaine : au même titre que le sexe, le sommeil ou le fait de manger; et cela a aussi été une dimension décisive de notre évolution. L'usage des outils est fondamentalement - avec le langage et

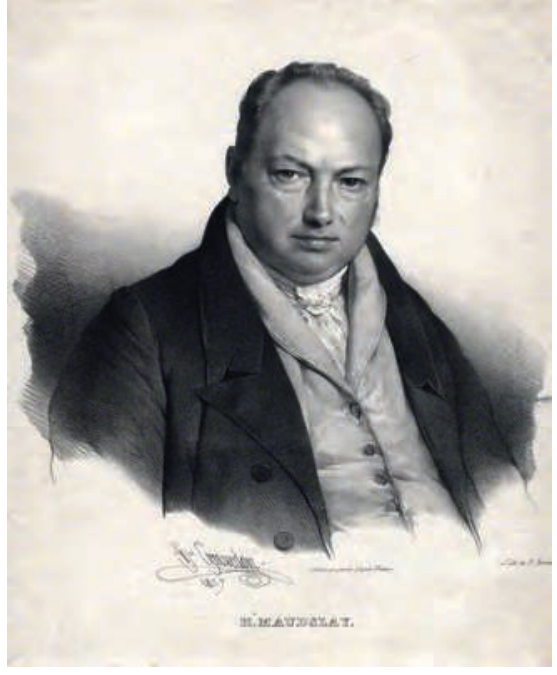

2. Henry Maudslay

d'après Pierre Louis (« Henri ») Grevedon, lithographie de Charles Étienne Pierre Motte, 1827 la station debout - ce qui fait de nous des humains. Des théories compliquées ont été avancées pour expliquer pourquoi nous avons adopté la station debout mais elles sont largement inutiles. Nos mains sont simplement devenues tellement utiles pour tenir des outils qu'il nous fallait nous en dispenser pour marcher.

Le premier grand singe connu pour avoir utilisé la bipédie est Lucy, il y a 3,7 millions d'années. Le premier « humain » véritable - Homo Habilis, date de prés de 2 millions d'années. Les premières civilisations et le langage écrit sont apparus, il y a seulement une centaine de milliers d'années, après que le cerveau humain a doublé de volume par rapport à celui d'Homo Habilis et quadruplé par rapport à celui de Lucy. Il a bien fallu un avantage évolutionnaire décisif pour expliquer un tel développement de la taille du cerveau. Francis pense que la coordination entre l'œil et la main ainsi que l'usage des outils ont joué ce rôle.

De puissants ordinateurs ont encore les plus grandes difficultés à attraper une balle, alors que cela ne nous pose aucun problème. Et même si on ignore tout de la technique on sait bien qu'on ne peut pas plier du verre, qu'on ne pousse pas une corde mais qu'on la tire, etc. On a tous un sens plus ou moins inné des matériaux. On utilisera, par exemple, un bâton pour ôter de la boue de ses chaussures, pour écarter une vache, pour faire tomber une pomme et pour toutes sortes de choses. Ces manières spontanées d'utiliser des outils sont tellement intuitives que nous n'en sommes pas conscients. Elles n'en sont pas moins inventives. D'ailleurs, si l'on en croit Francis, ce sont précisément des comportements de ce genre qui sont à l'origine de notre créativité.

Ses idées lui sont venues en enseignant, à de jeunes ingénieurs, le rôle joué par Henry Maudslay, cet artisan qui a inventé pratiquement à lui seul toutes sortes de machines outils au début du XIX ${ }^{e}$ siècle. Il a fabriqué, entre autres choses, une machine à faire des vis qu'il a utilisée d'abord pour faire des pas de vis sur ses propres tours, puis que d'autres compagnies ont employé par la suite pour faire les leurs. Les pas de vis que l'on trouve aujourd'hui sur tous les tournevis, mais aussi les 


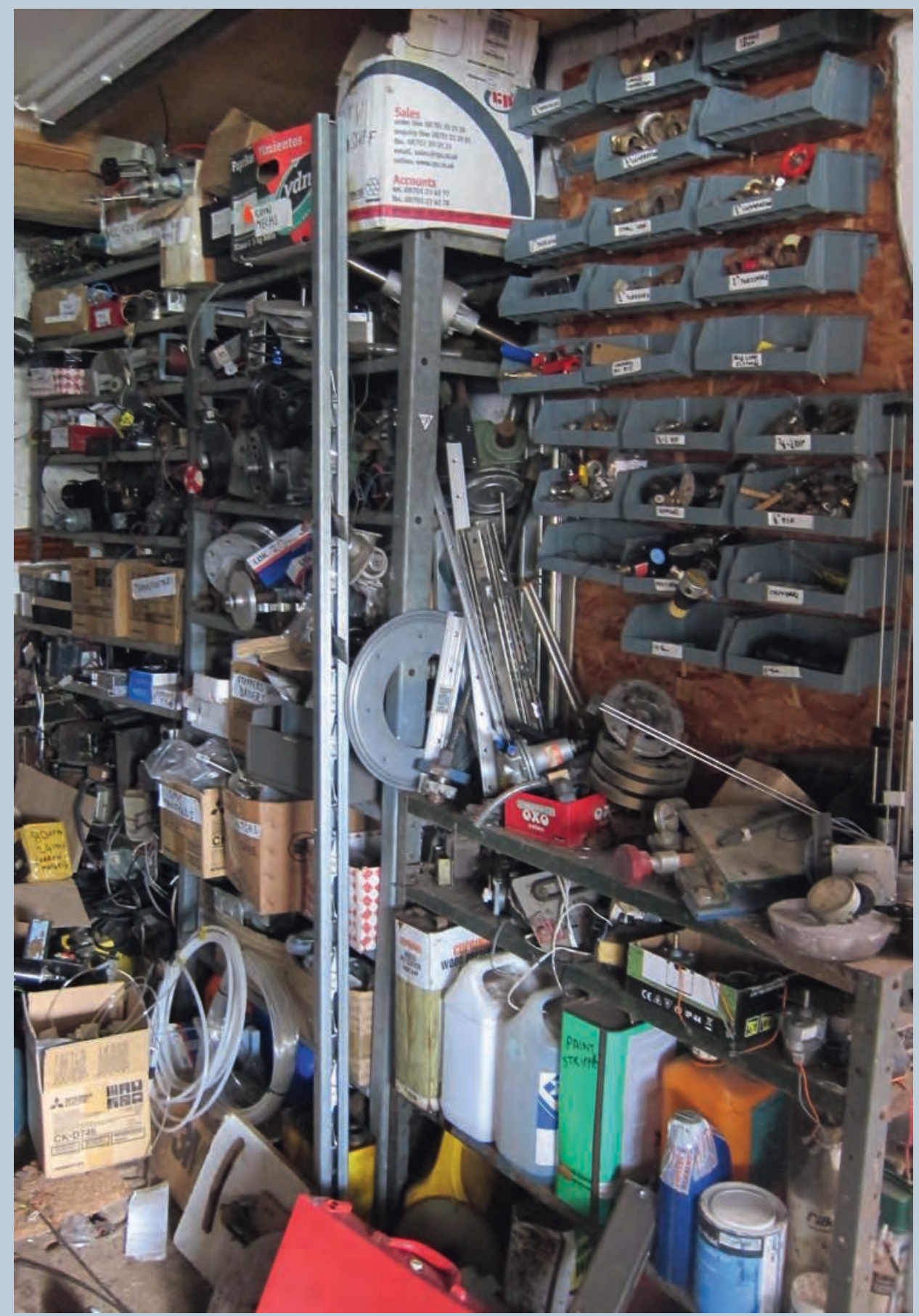

3. L'atelier de Tim Hunkin 

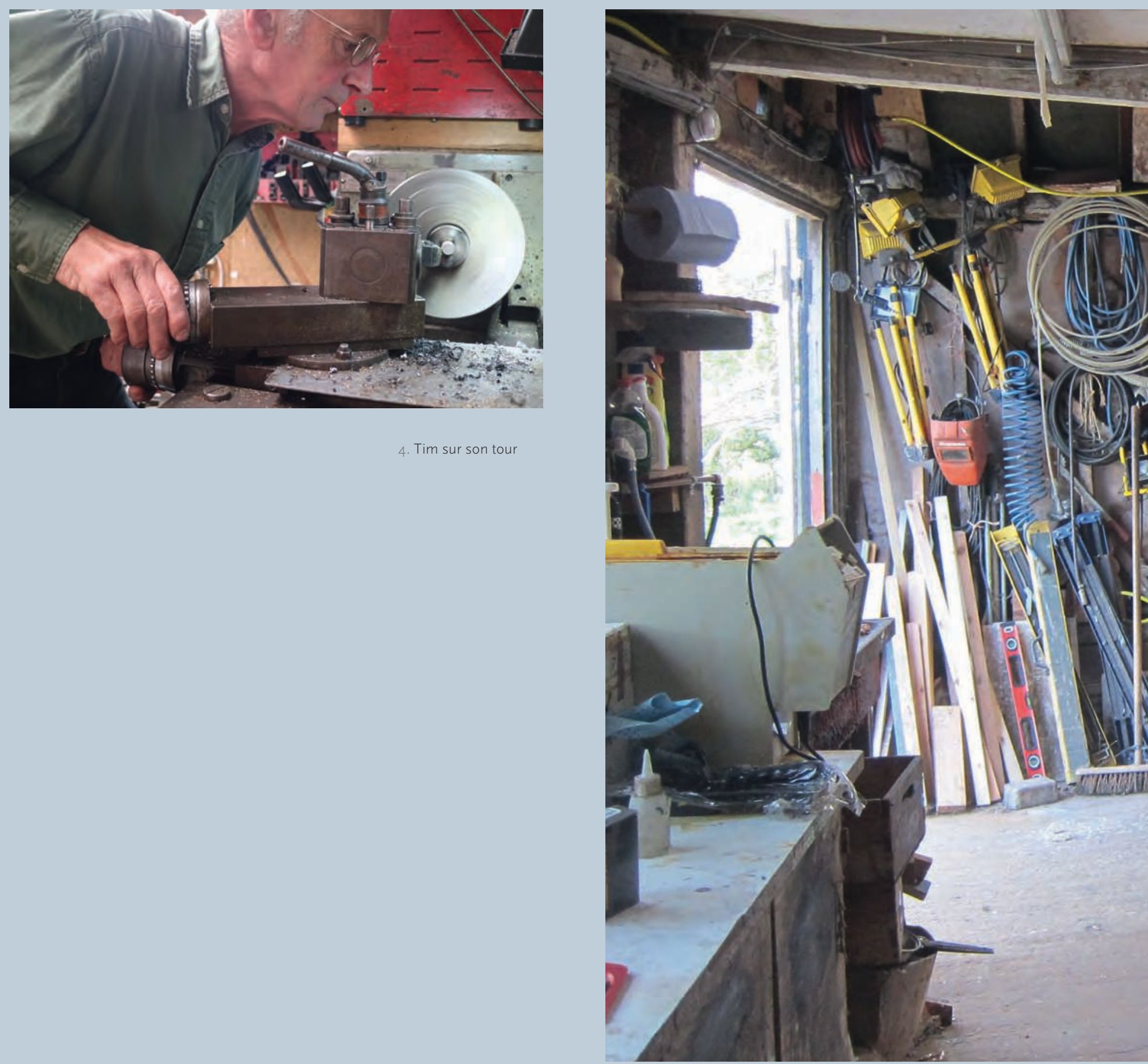

5. L'atelier de menuiserie 


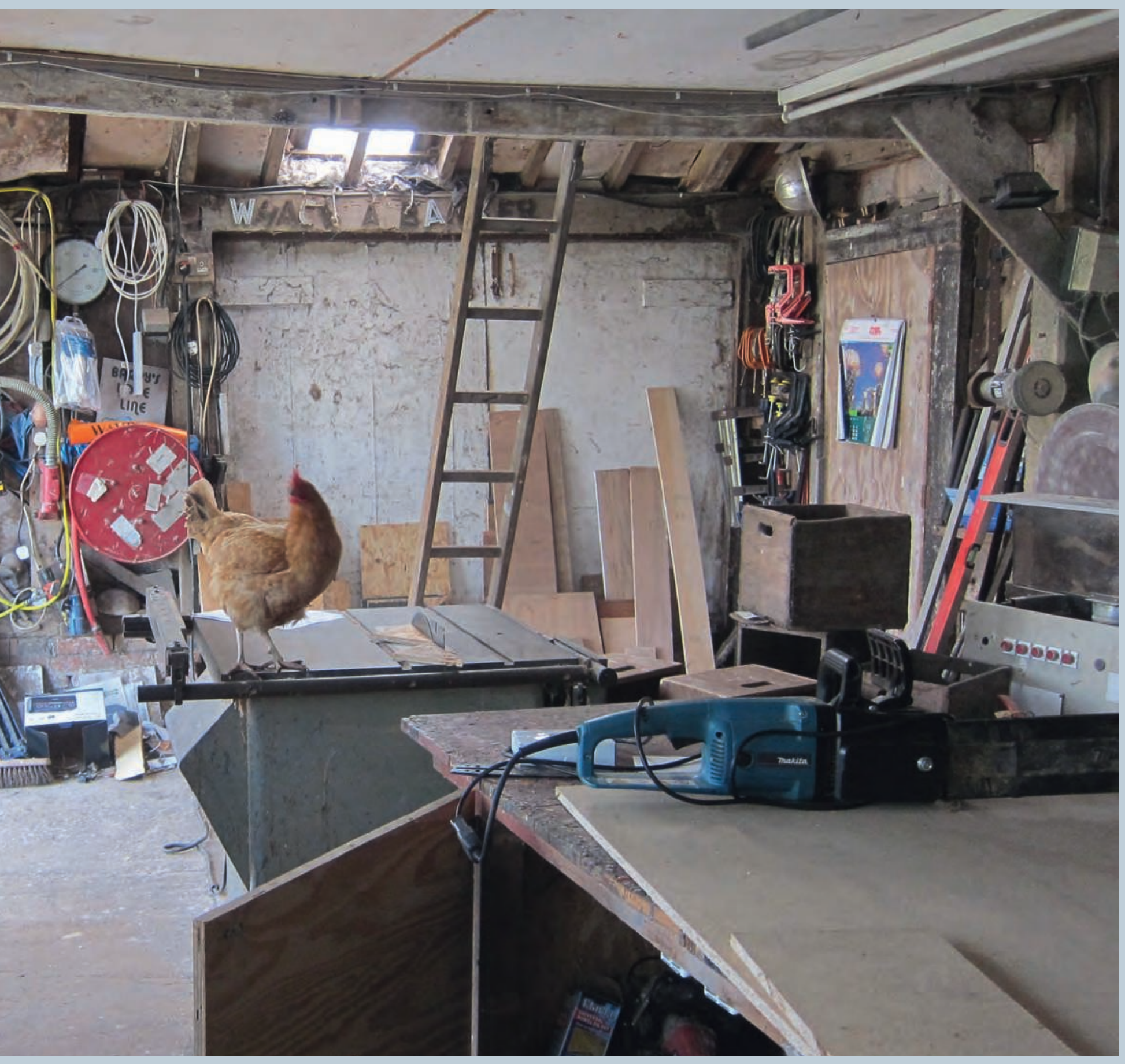


vis et les boulons proviennent de là. C'est en cherchant à comprendre l'inventivité à l'œuvre dans les machines-outils de Maudslay que Francis s'est mis à réfléchir aux origines de la technique.

Son papier n'a pas eu beaucoup de succès dans le milieu universitaire. Les implications étaient pourtant considérables; mais le style était anecdotique et il ne s'inscrivait dans aucune discipline établie. Je sais bien que l'on peut critiquer le détail de son argumentation. Mais cela n'enlève rien à son intuition centrale. Francis connaissait Richard Gregory, le célèbre psychologue. Richard pensait, je le sais, qu'il y avait beaucoup de vrai dans ce papier.

J'étais enthousiasmé par ses idées. Elles me semblaient tellement évidentes que je me demandais pourquoi elles n'étaient pas évidentes pour tous. Elles collaient aussi parfaitement à ma façon de penser et me permettaient de mieux la comprendre. J'aime bricoler depuis que je suis tout petit. Toute mon éducation m'incitait à me tourner vers des activités plus intellectuelles. Mais rien ne me semblait plus satisfaisant que de fabriquer quelque chose. Le point de vue de Francis me semblait parfaitement raisonnable. Il marrive parfois, sans même y penser, de fabriquer une pièce de machine tout à fait différemment de ce que j'avais envisagé. Quand j’ai mes outils et des matériaux devant moi, ce sont bien souvent mes mains qui décident. Et puis, je suis incapable de rester assis pour un certain temps sans faire quelque chose avec mes mains. Ce n'est pas un processus conscient, tel le moine qui manipule son chapelet. Les théories de Francis expliquent bien ce réflexe : une partie basique du fonctionnement de notre cerveau nous pousse à apprendre à faire des choses avec nos mains et à jouer avec.

Jusqu'à ce que je lise le papier de Francis, j'ai supposé que j'étais un dinosaure, quelqu'un qui en serait resté au xixe siècle. Mon atelier est une grange pittoresque avec un toit de chaume, emplie jusqu'à ras bord de morceaux de bois, d'outils et de bouts de métal. Il y en a des neufs que j'ai commandés dans des catalogues, et d'autres tout usés, récupérés dans des décharges et des vide-greniers. Je travaille avec Graham, un électricien qui en a eu assez de travailler de 9 à 5 et qui touche à tout. Des hirondelles viennent, chaque été, faire leur nid dans ma grange ; c'est pourquoi je ne peux jamais fermer la porte. Et puis il y a le chat de mon voisin, sourd et rouquin : il s'installe toujours dans un panier au centre de ma table de travail. Je dois l'avouer: ces lieux rappellent une autre époque.

Pourtant, le papier de Francis m’a fait comprendre que mon atelier et mes méthodes de travail n'étaient pas obsolètes et m'a incité à écrire ce qui suit. Je vois désormais mon lieu de travail différemment et de manière bien plus flatteuse, je dois l'avouer. Ma façon de travailler avec mes mains me paraît désormais plus fondamentale que toute activité scientifique ou artistique. C'est vrai que si je travaille de la sorte c'est d'abord parce que cela me plaît. Mais si je compare ma manière de faire avec la succession d'étapes, de dessins et de prototypes par laquelle on passe habituellement quand on fabrique des machines, la mienne me semble formidablement efficace. Nous avons mis quatre mois seulement avec Graham pour fabriquer la nouvelle horloge du zoo de Londres. Je vais à présent expliquer la méthode qui nous a permis d'être aussi efficaces.

Je fais des croquis de presque toutes les pièces que je fabrique. Ces esquisses rapides n'ont rien à voir avec les dessins précis des ingénieurs. Je change d'avis tout le temps et mon dessin n'arrête pas de changer, je le refais tout le temps. Dessiner de cette façon est une manière de 


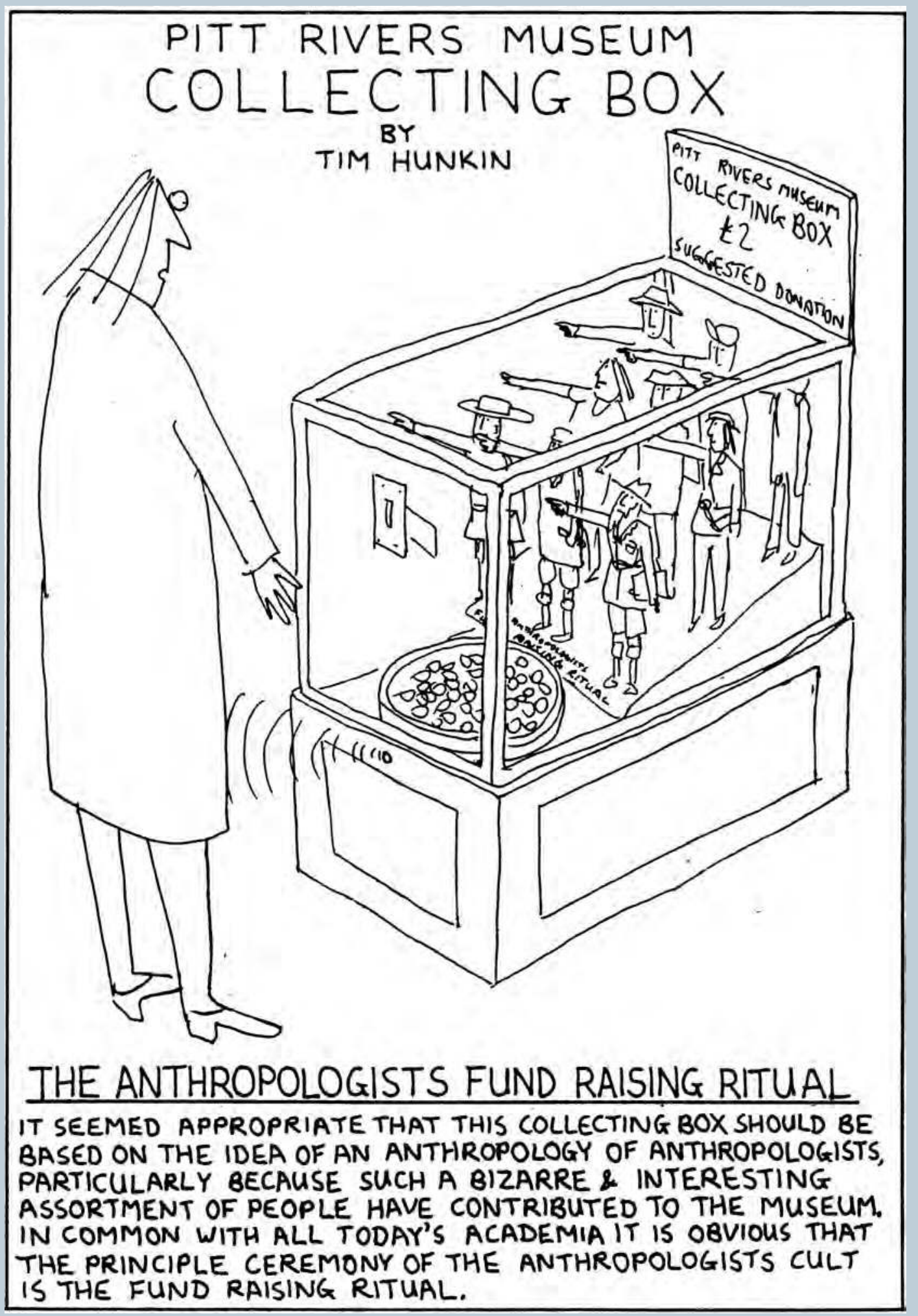




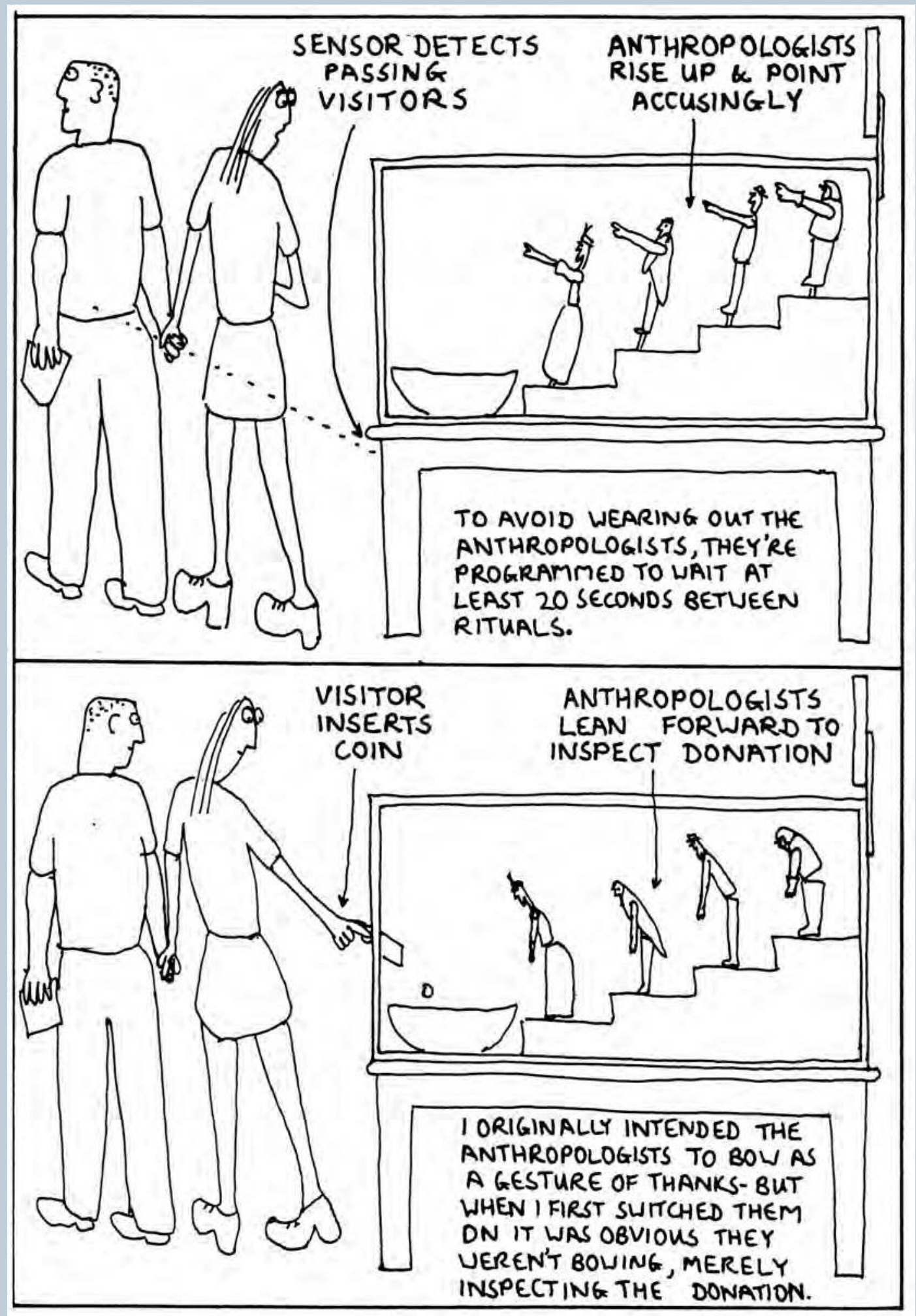




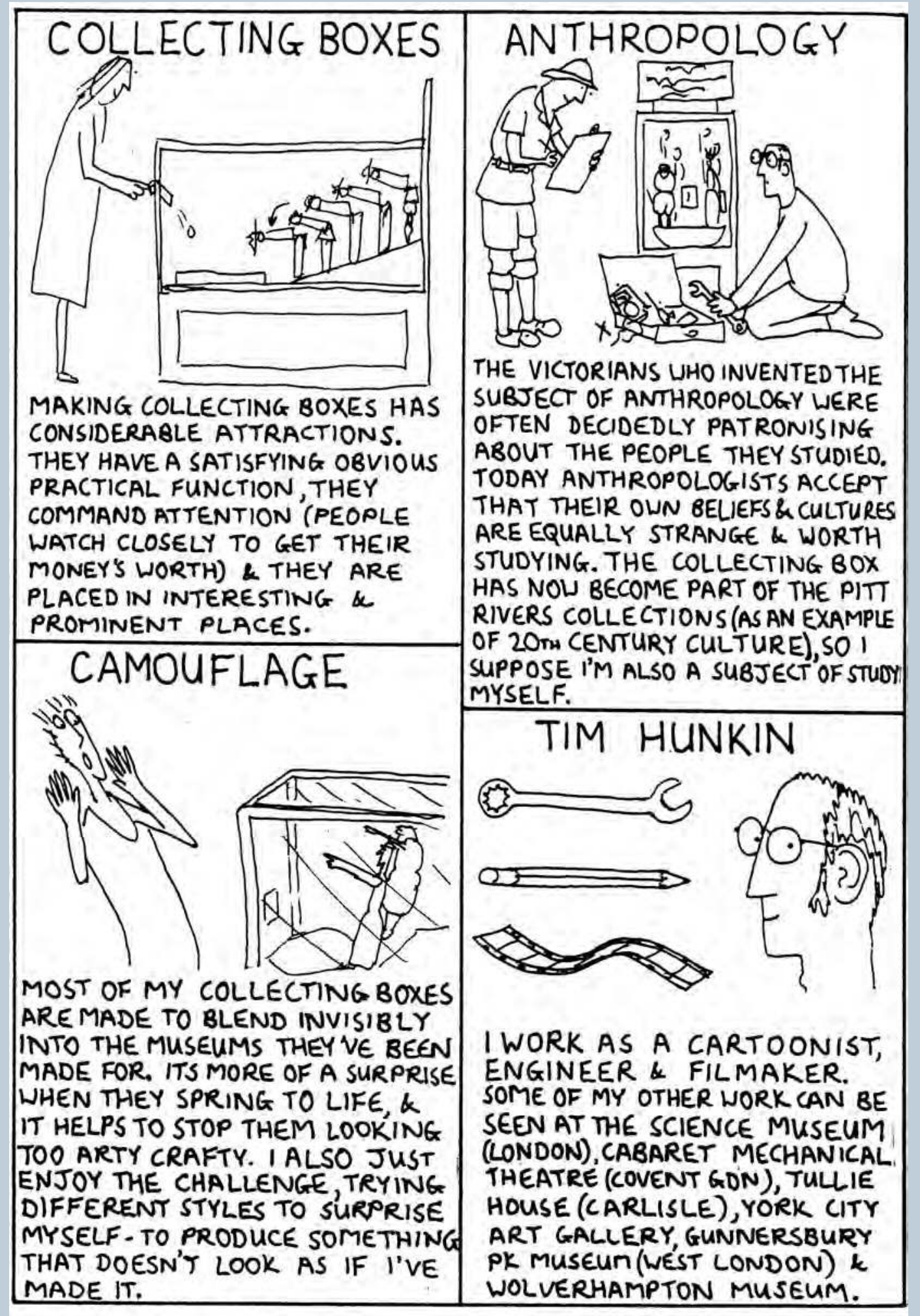


penser, cela permet de trouver les bonnes solutions et de rejeter les mauvaises. J'utilise aussi Solidworks, et je vois bien le bénéfice que l'on peut tirer à se servir de logiciels de dessin assisté (DAO) ; mais je préfère dessiner. En fait, il n'y a pas de vraie différence entre dessiner les pièces d'un objet qu'on fabrique et faire une caricature. On refait sans cesse son dessin, on essaie de rendre une idée aussi claire et incisive que possible ; on pense aussi continuellement à d'autres possibilités pour améliorer ce que l'on est en train de faire.

Savoir dessiner les pièces d'une machine dépend de l'expérience dont on dispose. Je n'ai pas souvenir d'avoir dessiné les machines que je faisais quand j'étais enfant ; et quand j'ai recommencé à en faire après avoir terminé mes études à Cambridge, je faisais très peu de dessins, j'améliorais les détails en utilisant la méthode par essais et par erreurs. Il y a tellement de facteurs en jeu, surtout quand on conçoit les pièces mobiles d'une machine : un dessin ne servait pas à grand-chose. Il s'agissait de savoir si un levier était suffisamment rigide, si un ressort faisait contrepoids, si une vis permettrait de retenir une poulie sur un arbre de transmission. Est-ce qu'un moteur sera assez puissant? Est-ce qu'il s'arrêtera suffisamment vite? La seule façon de le savoir était d'essayer et de faire usage de ses sens (le son et les odeurs sont bien utiles parfois pour identifier un problème). Tout essayer par soi-même prend cependant un temps fou. Avec l'expérience, il est plus rapide de résoudre des problèmes de conception sur le papier.

Dessiner les pièces d'une machine nécessite des informations de départ fiables. Quand je fabrique une machine, je dessine systématiquement une pièce et la fabrique immédiatement avant de dessiner la suivante. J'ai un vague plan d'ensemble, mais je préfère construire chaque pièce l'une après l'autre. Chaque pièce terminée détermine la suivante. Je trouverais cela trop contraignant de concevoir en détail une machine dans sa totalité avant même de commencer à la fabriquer ; j'aurais peur d'improviser, comme c'est devenu le cas de beaucoup d'ingénieurs aujourd'hui. On prend généralement de meilleures décisions si on opère par tâtonnement, c'està-dire, essentiellement, en commençant par des prototypes. Dans mon atelier, j'ai tout ce dont j'ai besoin autour de moi ; je peux ainsi passer sans effort du dessin au prototype.

Cet atelier rempli de toutes sortes de pièces - particulièrement les coins où sont accumulées celles récupérées ici ou là - joue un rôle fondamental dans ma manière de procéder. En examinant tout ce que j'ai en stock, je découvre souvent de nouvelles façons de fabriquer une pièce et je peux aussi réutiliser parfois quelque chose dont je dispose déjà. Tout ce que j’ai en stock - et mes outils aussi, bien sûr - constitue une véritable extension de mon cerveau ; c'est ma mémoire matérielle. Et lorsqu'il me manque quelque chose, je peux consulter la vaste collection de catalogues dont je dispose. De vieilles granges de campagne comme la mienne peuvent sembler démodées au premier abord ; mais elles ne sont certainement pas coupées du monde. Les moyens de distribution moderne me permettent d'obtenir tout ce que je veux en 24 heures. Mes voisins sont sidérés par le flux constant de camions de livraison qui passent devant chez eux.

Le fait de fabriquer soi-même les pièces d'une machine peut sembler un travail lent et répétitif. Mais c'est du temps bien dépensé. D'abord parce que c'est satisfaisant de travailler avec ses mains. Je ne suis pas un grand artisan mais j'ai plus de plaisir à faire des soudures nettes avec ma merveilleuse machine à souder au tungstène (TIG : Tungsten Inert Gas ; procédé de soudure 
à l'arc) que j'en ai à travailler avec n'importe quel programme d'ordinateur. Ensuite, à la fin de chaque journée de travail, j'ai la satisfaction de savoir que j'ai réalisé quelque chose de concret. C'est excellent pour le moral quand on s'y remet le lendemain ; même si c'est pour s'apercevoir, en fin de compte, qu'il faut recommencer tout ce qu'on a fait la veille. Enfin, cela me donne du temps pour réfléchir à l'étape suivante ou pour améliorer ce que je suis en train de fabriquer ; parfois, même, cela me donne de nouvelles idées pour la machine dans son entier.

Il est étrange de constater l'écart qui se creuse chaque jour davantage entre les procédés de fabrication employés dans les pays industrialisés et ma façon de travailler avec les mains ; mais, plus généralement aussi, avec toute activité pratique. Surtout quand on en mesure l'efficacité et qu'on réalise à quel point cela fait partie de notre nature. Le savoir-faire pratique est certainement considéré comme inférieur au talent « intellectuel ». Mais cela n'a aucun sens. N'est-ce pas le dictionnaire Chambers qui définit l'intellect comme l'« usage des capacités rationnelles de l'esprit ». Or, si on en croit une telle définition, on ne peut douter que je fais usage de mon intellect dans mon atelier. On trouve différentes définitions d'un intellectuel dans le dictionnaire mais celle que je préfère est la suivante : « une personne d'intelligence ou de savoir supérieur », ce qui suggère souvent un doute quant à sa sagacité (ou son sens) pratique. En d'autres termes, un intellectuel est simplement quelqu'un d'incapable de faire quoi que ce soit de pratique.

Francis a souvent dit que « la technique est trop importante pour en laisser la responsabilité à des ingénieurs », et je partage son point de vue. Mon diplôme en sciences de l'ingénierie m'a donné l'impression qu'on pouvait appliquer une approche scientifique à n'importe quel problème d'ingénierie et que toute forme de savoir pratique, acquise de manière intuitive, était obsolète. Il n'existait aucune idée de la complémentarité des deux approches. À présent, je vois cela comme du snobisme. Dans l'école publique où j’ai étudié, on considérait les études d'ingénieur comme une voie adaptée aux élèves pas assez brillants pour faire des sciences et je suis sûr que les responsables de l'école d'ingénieurs de Cambridge cherchaient à prouver que leur domaine était aussi académique que les autres.

Rien de bien nouveau, d'ailleurs. Les ingénieurs ont toujours bataillé depuis le xviI siècle pour se voir reconnaître un statut plus élevé. Au xviII siècle, le mot « ingénieur » servait à désigner ceux qui construisaient ou qui exploitaient les engins utilisés dans les mines. Mais Jame Naysmith, l'ingénieur qui inventa au xixe siècle le marteau-pilon (à vapeur) a écrit : « il vaut mieux se servir de ses yeux et de ses mains - tout particulièrement de ses mains nues - pour savoir ce que l'on fait et bien connaître les matériaux qu'on utilise, trop de gens portent des gants. Les gants, tout particulièrement les gants en chevreau, représentent précisément tout ce qu'un ingénieur doit éviter... En conséquence : je n'ai aucune confiance dans ces jeunes ingénieurs qui se vantent de leur savoir technique ». Cette citation acquiert une résonance toute particulière, aujourd'hui, quand on sait que le port de gants est devenu obligatoire sur les chantiers.

On ne peut manifestement pas concevoir de projets à grande échelle ou qui mettent en jeu des techniques de pointe et employer les mêmes méthodes que celles que j'utilise dans ma grange. Mais si j'en crois mon expérience, plus rien, en réalité, n'est fait de cette façon. On considère simplement que cela est démodé et inefficace. Les salles d'activités interactives que l'on trouve dans les 
Musées des sciences, autrefois construites pour des essais ou en cas d'erreurs d'expérimentation, sont à présent plus ou moins exclusivement conçues sur le papier, et utilisées pour la fabrication seulement après que tous les détails ont été réglés. Leurs concepteurs tendent aujourd'hui à être des diplômés en sciences de la communication, démunis de savoir pratique et qui trouvent trop coûteux de faire des prototypes. Les gens qui travaillent dans mon laboratoire local de BT Martlesham me disent constater le même phénomène. On ne fait plus que du software («logiciel ») dans ce centre d'innovation important. Il n'y a plus un seul atelier. Graham, qui travaille avec moi, a précisément quitté son travail précédent dans une compagnie spécialisée dans les automates industriels parce que cela le déprimait de constater la manière dont son travail avait évolué. Bien loin de pouvoir faire preuve de son savoir-faire, il devait suivre aveuglément les dessins qu'on lui donnait. Je suis sûr qu'il existe encore des domaines où les choses sont conçues de manière traditionnelle. Mais les entreprises préfèrent ne pas en parler, et valorisent uniquement les techniques de pointe développées lors du travail de conception.

Les élèves ingénieurs d'aujourd'hui ne doivent pas seulement digérer toutes les sciences, il leur faut suivre également un enseignement théorique en gestion ; c'est à croire que les écoles d'ingénieurs veulent abandonner leur cœur de métier. J'ai rencontré à ce sujet le directeur des enseignements à l'Académie des sciences, il y a quelques années. Il est resté poli, mais il pensait manifestement que j'étais un excentrique et un marginal pour oser suggérer que leur approche de la technique était trop restrictive.

Le cour du problème est qu'on a longtemps disposé d'une abondance de travailleurs qualifiés formés comme apprentis et qui avaient acquis intuitivement un vrai savoir-faire. La plupart passaient leur vie à effectuer des tâches physiques répétitives et exténuantes mais une minorité jouait un rôle indispensable pour résoudre les problèmes pratiques qui se posaient aux élites. Les universités et les collèges d'art avaient ainsi recours à des techniciens qualifiés capables de résoudre des problèmes pratiques et d'enseigner ces savoir-faire aux étudiants de manière informelle, sans attendre de reconnaissance. D’ailleurs, je plaide coupable aussi : j’ai dessiné plusieurs pièces de machine, l'an dernier, avec un logiciel d'assistance par ordinateur (DAO) que j'ai envoyé à un fabricant local. Mais ce que j’ai dessiné n’a pu marcher que parce que Mark, le chef soudeur, modifiait intuitivement mes dessins un peu trop « impressionnants ». Il procède d'ailleurs de la sorte avec tout ce qu'on lui demande de faire.

Les gens comme Mark sont extrêmement brillants. J'enseignais à des gens comme lui, dans un cours de l'Open University intitulé « Dessin et innovation». Mes étudiants étaient en majorité des ingénieurs compétents sur le plan pratique mais désireux d’obtenir un diplôme. Ma plus grande satisfaction était de les voir gagner en confiance. Leur crainte initiale vis-à-vis de leurs tuteurs et du système universitaire diminuait progressivement et ils finissaient par questionner tout ce qu'on leur enseignait.

Tant qu'il y avait cette abondance d'artisans qualifiés, personne n'aurait accepté de considérer les savoir-faire pratiques comme des choses de valeur. Mais le monde industrialisé a bien changé. Il y a maintenant moins de formations d'apprentis que de places à l'Université. La masse des emplois se situe dans les industries de service - industrie alimentaire ou centres d'appel ; on ne la trouve 
plus dans le travail manuel. Je pensais récemment encore que l'absence de corrélation entre travail manuel et emploi de masse aboutirait à faire respecter d'avantage les compétences pratiques. Malheureusement, l'afflux d'ouvriers qualifiés en provenance de l'Europe de l'Est a permis au Royaume-Uni de maintenir l'illusion que les savoir-faire pratiques étaient toujours bon marché, même s'il est évident que nos compétences se sont dramatiquement atrophiées dans ce domaine.

Les écoles et les collèges se débarrassent de leurs ateliers consacrés à des activités et à des formations pratiques. Les disciplines à caractère plus académique sont bien plus commodes, elles ne demandent pas d'équipement ou de matériaux coûteux ; et elles ne posent pas de problèmes en termes de santé ou de dangerosité. De plus, le gouvernement insiste pour envoyer tout le monde à l'Université. Pourtant les enseignants sont fascinés par l'enthousiasme que montrent les enfants - souvent d'ailleurs les plus indisciplinés - lors d'ateliers pratiques comme ceux qu'organise ma femme. En réalité, nombre d'enfants brillants sont rebutés par les sujets académiques. Mais il faut dire aussi que la plupart d'entre eux sont peu encouragés à faire quoi que ce soit avec leurs mains chez eux, si bien que l'organisation de travaux pratiques se fait chaque jour plus difficile : la plupart des enfants sont aujourd'hui incapables d'utiliser le moindre outil, pas même des ciseaux.

Les comptables ne comprennent rien au travail d'ingénieur. Il y a quelques années de cela, ils ont convaincu la plupart des compagnies de se débarrasser de leurs magasins d'usine et de leurs stocks, parce qu'ils étaient difficiles à comptabiliser et qu'ils étaient démodés. Mais cela nuit à l'efficacité du travail des ingénieurs parce que faute de disposer de ce dont ils ont besoin sur place, ils sont obligés de tout acheter et d'attendre indéfiniment la livraison de ce qu'ils ont commandé. C'est extrêmement coûteux, en comparaison du prix dérisoire retiré de la vente de leurs anciens stocks.

Les journalistes comme les critiques ou les politiciens travaillent d'abord avec des mots. Ils ne comprennent pas la nature de l'activité pratique. Il y a quelques années, durant la période de frénésie que nous avons connue à propos du prix des plombiers, les médias insistaient sur le fait que tout le monde pouvait se

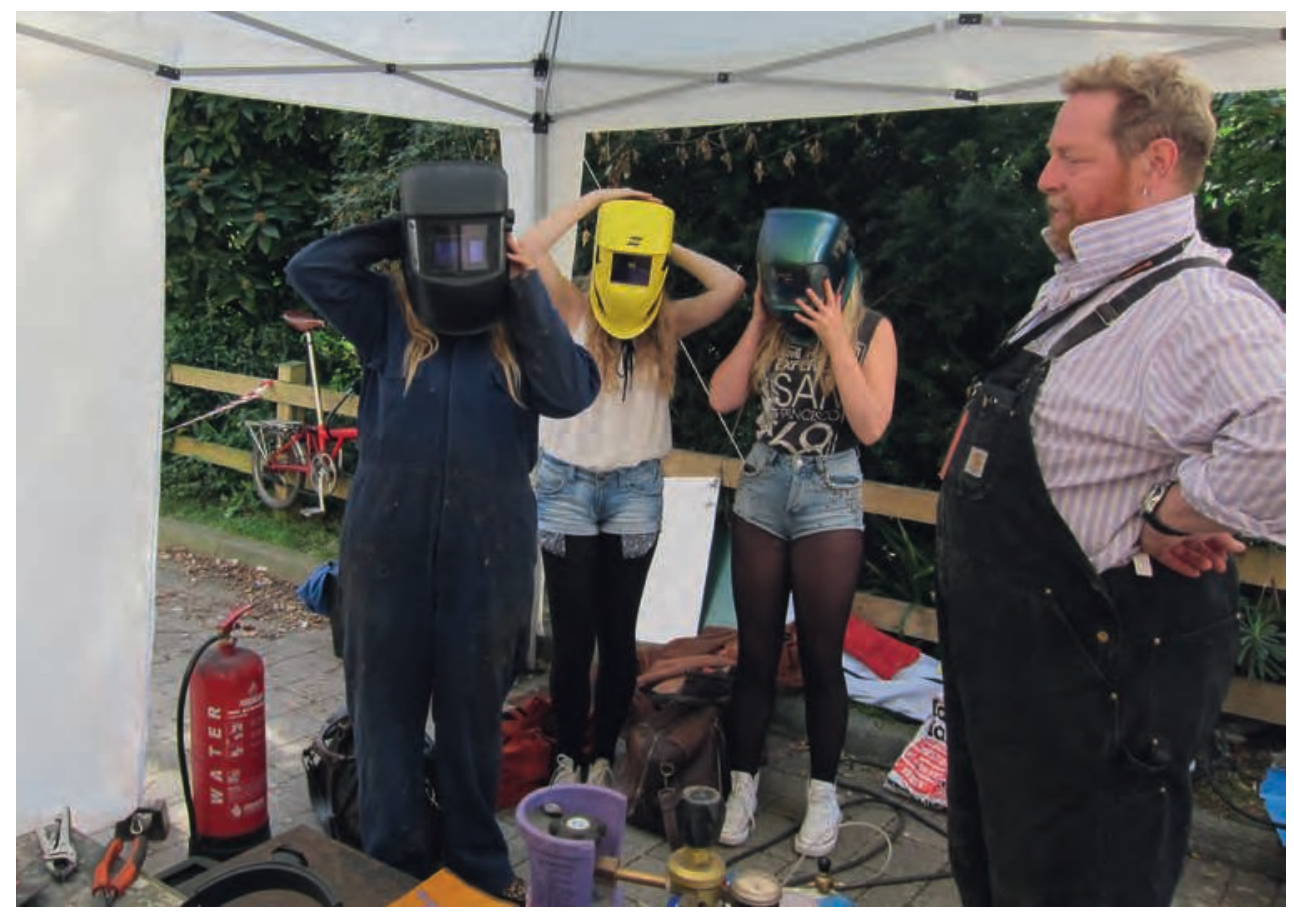


reconvertir et faire de la plomberie. C'était insultant pour les plombiers. Il faut plusieurs années, en réalité, pour acquérir un savoir pratique comme le leur. Mais les ingénieurs, les concepteurs ou les travailleurs qualifiés ne savent pas vraiment valoriser leur pratique parce que leurs compétences véritables ne sont pas d'ordre verbal. Je me sens moi-même vulnérable en rédigeant ce texte; je suis bien plus confiant quand je fabrique des objets.

Il y a quelques décennies, l'apprentissage traditionnel prenait cinq ans et c'était le meilleur moyen d'acquérir un savoir d'ordre pratique. Privé de la base industrielle dont disposait autrefois la Grande-Bretagne, il n'est plus possible aujourd'hui de ranimer le réseau des apprentissages à grande échelle. Existent heureusement d'autres façons d'apprendre. Je n'ai jamais suivi d'apprentissage mais suis né avec la capacité de bricoler et j'ai appris par moi-même à faire des choses avec mes mains, en m'inspirant aussi de Rex Garrod ou d'autres. Cela a été lent. Il m’a fallu une quinzaine d'années avant de pouvoir en vivre. Mais c'est possible. D’autres personnes de ma connaissance ont été inspirées par des parents ou des grands-parents doués de sens pratique. Et je suis sûr aussi qu'il y a d'autres moyens d'apprendre auxquels je n'ai pas pensé. Beaucoup de compétences pratiques sont plus aisées à acquérir aujourd'hui que par le passé car de nombreux outils sont moins chers et plus faciles à utiliser. Je suis optimiste à long terme. La rareté croissante des compétences pratiques va conduire à ce qu'elles bénéficient d'un plus grand respect et qu'elles acquièrent un statut plus élevé dans le futur. Elles reviendront aussi sous de nouvelles formes. C'est par exemple seulement après que la plupart des gens ont cessé de produire leur propre nourriture au xixe siècle que le jardinage est devenu un passe-temps à la mode.

Je peux me faire des illusions mais je crois détecter un changement d'attitude. Les étudiants en art graphique avec qui je discutais en décembre au Royal College of Arts voulaient désespérément échapper à leurs programmes informatiques. L'intérêt actuel de la communauté scientifique pour le physical computing reflète la même tendance. La popularité de programmes de télévision comme le scrap heap challenge (jeu d'ingénierie télévisé produit par RDF Médias et diffusé sur Channel 4 au Royaume-Uni) - bien qu'ils ne m'enthousiasment pas vraiment - dénotent quand même un regain d'intérêt pour la technique appliquée. D’ailleurs, sur la Côte Ouest des États-Unis, les gens qui s'intéressent à la technique se définissent eux-mêmes comme des bricoleurs (tinkerers); et le mot n'a pas, là-bas, les connotations d'incompétence et d'amateurisme qu'il peut encore avoir ici. Le magazine "Make » dédié aux bricoleurs de la Côte Ouest est bien établi et organise chaque année une fête formidable. Ce qui réunit est le plaisir commun de jouer avec la technique. Même Microsoft en a reconnu le potentiel et y tient un pavillon entier (légèrement rasoir, d'ailleurs).

Je suis vraiment ravi de ce que je fais. Jouer avec la technique est vraiment ce qui me motive. Quand je lis les textes pompeux et soi-disant « conceptuels » associés à l'art contemporain ou encore quand je constate à quels détails infimes s'intéressent aujourd'hui les sciences fondamentales pour comprendre le monde, je comprends à quel point j'ai de la chance de m'être investi ainsi dans la technique. On réalise encore à peine, aujourd'hui, le formidable potentiel que recèle l'art de combiner le travail traditionnel des ingénieurs avec l'informatique. 


\section{Notes}

1. Pour se faire une idée relativement détaillée de l'œuvre protéiforme de Tim Hunkin, il n'y a pas de meilleure idée que de prendre le loisir de visiter son site. On ne le regrettera pas : http://www.timhunkin.com/.

\section{Traduction}

La rédaction de Techniques\& Culture tient tout particulièrement à remercier Denis Vidal pour son travail de traduction, l'intérêt et la générosité de ses entretiens avec Tim hunkin.

\section{I'auteur}

Tim Hunkin est un ingénieur anglais, réalisateur de dessins animés, auteur et artiste qui vit dans le Suffolk, en Angleterre. Il est connu pour avoir créé pour Channel 4, la série télévisée La Vie Secrète des Machines, dans laquelle il explique l'histoire et les dispositifs divers de l'environnement ménager. Il a aussi conçu des expositions pour les institutions et les musées au Royaume-Uni et organisé de nombreux ateliers publics de construction mécanique, principalement destinés au divertissement.

\section{Iconographie}

Image d'ouverture. (C) Tim Hunkin.

1. Dessins, Tim Hunkin.

2. (C) National Portrait Gallery, London.
3 à 6. (C) Tim Hunkin.

Planches. Pitt Rivers Museum « Collecting Box». The Anthropologists Fund Raising Ritual par Tim Hunkin.

\section{Pour citer l'article}

Hunkin, T. 2017 «La technique à visage humain. "Technology is what makes us human" ", Techniques\& Culture 67 « Low tech? Wild tech!», p. 64-81. 